\title{
Mightability: A Multi-State Visuo-Spatial Reasoning for Human-Robot Interaction
}

\author{
Amit Kumar Pandey and Rachid Alami
}

Authors are with CNRS; LAAS; 7 avenue du Colonel Roche, F-31077 Toulouse, France. Université de Toulouse; UPS, INSA, INP, ISAE; LAAS; F-31077 Toulouse, France.

Email: akpandey@laas.fr and rachid.alami@laas.fr

We, the Humans, are capable of estimating various abilities of ourselves and of the person we are interacting with. Visibility and reachability are among two such abilities. Studies in neuroscience and psychology suggest that from the age of 12-15 months children start to understand the occlusion of others line-of-sight and from the age of 3 years they start to develop the ability, termed as perceived reachability for self and for others. As such capabilities evolve in the children, they start showing intuitive and proactive behavior by perceiving various abilities of the human partner.

Inspired from such studies, which suggest that visuo-spatial perception plays an important role in Human-Human interaction, we propose to equip our robot to perceive various types of abilities of the agents in the workspace. The robot perceives such abilities not only from the current state of the agent but also by virtually putting an agent into various achievable states, such as turn left, stand up, etc. As the robot estimates what an agent might be able to 'see' and 'reach' if will be in a particular state, we term such analyses as Mightability Analyses. Currently the robot is equipped to perform such Mightability analyses at two levels: cells in the 3D grid and objects in the space, which we termed as Mightability Maps (MM) and Object Oriented Mightabilities (OOM) respectively.

We have shown the applications of Mightability analyses in performing various cooperative tasks like show and make an object accessible to the human as well as competitive tasks like hide and put away an object from the human. Such Mightability analyses equip the robot for higher-level learning and decisional capabilities as well as could facilitate the robot for better verbalize interaction and proactive behavior.

The work described here has been partially conducted within the EU Project CHRIS (Cooperative Human Robot Interaction Systems) funded by the E.C. Division FP7-IST under Contract 215805.

\section{Introduction}

We, the Humans, have the capability to perceive (imagine) various abilities of ourselves as well as of others. Interestingly we not only perceive such abilities of the current state of the person but also we imagine various states in which the person might be from her current state and roughly estimates her capabilities in that virtual state. As shown in fig.1(a), the person P2 wants the green bottle containing 

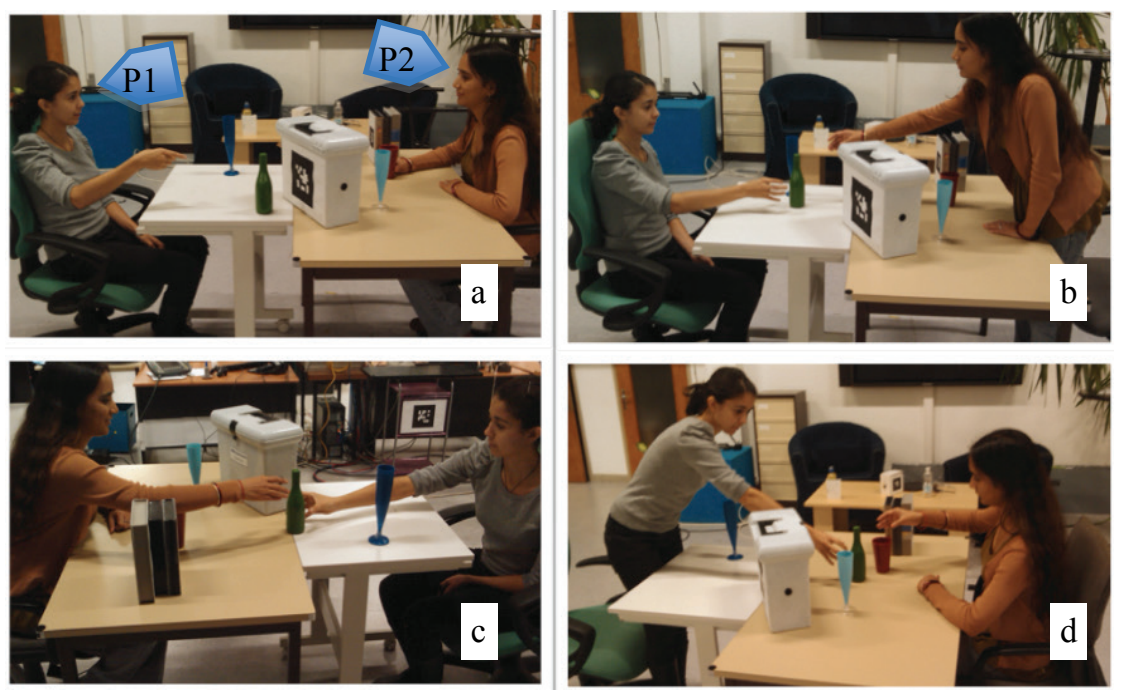

Fig. 1 Different ways, in which person P1 can make the green bottle accessible to the person P2.

drink. Depending upon various factors such as the current state, the relation, the comfort preferences, etc., the person P1 can make the bottle accessible in various ways: fig. 1(a), by verbally describing how to get the bottle e.g. "Stand up and you can see and get the bottle", fig. 1(b), by taking the bottle and putting it at a place visible to the $\mathrm{P} 2$ so that she can reach it by standing up and leaning forward, fig. 1(c), by putting the bottle at a place so that P2 can get it by leaning forward while remains seated, (d) by putting the bottle at a place so that $\mathrm{P} 2$ can get it just by simple arm motion. Of course there could be various other ways.

The key point is, in all situations the person $\mathrm{P} 1$ is able to perceive the reachability and visibility not only for herself but also of the person she is interacting with. More interestingly she predicts such abilities not only for the current states of both but also in different states like standing, leaning etc. Furthermore she also estimates the commonly visible and reachable places in different states.

In this paper we will focus on two abilities: Visibility and Reachability. Studies in psychology and neuroscience suggest that from the age of 3 years, children are able to perceive places reachable by them and by others, as the sign of early development of allocentrism capability, i.e. spatial decentration and perspective taking, [1]. At 12-15 months of age children show evidence of an understanding of occlusion of others line-of-sight $[2,3]$ as well as the concept of others not able to see 'behind' $[4,5]$. Evolution of such abilities of visuo-spatial reasoning in children enables them to help, co-operate and understand the intention of others.

Inspired from such evidences, we propose to equip our robots with such basic yet important capabilities of analyzing visuo-spatial abilities of itself and of the human partner. 
Reachable and manipulable workspaces have been represented in various ways. Offline techniques for mapping kinematic reachability and directional structure for the robot arm [6], and workspace to the configuration space for redundant manipulator [8], have been developed and used in generation of reachable grasp [7]. In [9], a Monte Carlo based randomized sampling approach stores the true or false information about the reachability of a cell. However none of these works focus on such analyses in different states as well as they don't calculate such abilities from the human's perspective, which is important for Human-Robot Interaction.

Visual reasoning, mainly focusing on perspective taking, has been addressed from the point of view of learning [10], action recognition [11] and shown useful in Human-Robot Interaction [12]. In [13], our robot analyses geometric visual perspective of the human partner. But these works don't take into account different possible states achievable by the robot and the human from their current states.

In this paper we will present the concept of Mightability, which stands for Might be Able to. Subsequently we will present various states, which the robot takes into account to estimates reachability and visibility. Then the two levels of abstraction for such Mightability Analyses will be presented: at the level of cells in a discretized 3D workspace, which we will call as Mightability Maps (MM) and at the level of objects in the workspace which we termed as Object Oriented Mightability (OOM). Section 3 will be dedicated to various applications of such Mightability Analyses such as finding the search spaces for different tasks as well as their potential use in learning semantics of a task, in generation of shared plan and in grounding changes to agents. Then we conclude with the pointer to various other potential applications and future works.

\section{Mightability : Might be Able to ...}

The motivation behind this work is to enrich the robot's knowledge about various abilities of itself and of the human partner not only from their current states but also of the different states, which they can achieve. Each such estimation answers to a particular question, e.g. "if the agent will turn around, which are the places he might be able to reach", "if the agent will stand up, which are the objects he might be able to see", etc. Below we discuss different possible states presently taken into account.

\subsection{Multi-State: Possible Achievable States}

For the current implementation the robot is already provided with the set of different states, which an agent might achieve as well as the corresponding virtual actions, which need to be performed for achieving those states. Various possible 


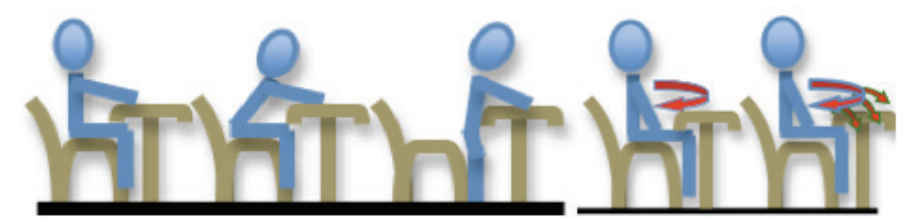

Fig. 2 (a)-(e) Taxonomy of reach actions (a) arm-shoulder reach, (b) arm-torso reach, (c) standing reach. We have augmented the set by (d) turning around reach and (e) turning around and leaning reach.

states for estimating reachability have been inspired from the studies of human movement and behavioral psychology $[14,15]$, which identifies different types of reach action of the human ranging from simple arm extension (arm-only reach), shoulder extension (arm-shoulder reach), leaning forward (arm-torso reach) and standing (standing reach), as shown in fig. 2(a)-(c). We have augmented various other reachability analyses to this set: turning-around reach, fig. 2(d), turningaround and leaning reach, fig. 2(e), for sitting as well as for standing postures.

Table 1 and table 2 show various states for reachability and visibility analyses the robot is performing for itself and for the human partner.

Table 1: Different states for Spatial-Reach Mightability analysis (reachability is calculated for only by left hand, only by right hand and by both hands)

\begin{tabular}{|l|l|}
\hline State & Virtual Action \\
\hline Current & - \\
\hline Leaning Forward & Lean Torso Forward \\
\hline Turning Right & Turn Torso Right \\
\hline Turning Left & Turn Torso Left \\
\hline Turning Right and Lean & Turn Torso Right and Lean Torso \\
\hline Turning Left and Lean & Turn Torso Left and Lean Torso \\
\hline Standing & Stand up \\
\hline Standing Lean & Stand up and Lean Torso \\
\hline Standing Turning Right Lean & Stand up, Turn Torso Right and Lean Torso \\
\hline Standing Turning Left Lean & Stand up, Turn Torso Left and Lean Torso \\
\hline
\end{tabular}

Table 2: Different states for Visual Mightability analysis

\begin{tabular}{|l|l|}
\hline State & Virtual Action \\
\hline Current & - \\
\hline Looking Straight Sitting & Turn Head Straight \\
\hline Looking Right Sitting & Turn Head Right \\
\hline Looking Left Sitting & Turn Head Left \\
\hline Looking Straight Standing & Stand up and Turn Head Straight \\
\hline Looking Left Standing & Stand up and Turn Head Left \\
\hline Looking Right Standing & Stand up and Turn Head Right \\
\hline
\end{tabular}




\subsection{Estimation while Respecting Postural and Environmental Constraints}

Robot performs Mightability Analyses by taking into account collision as well as the joint limits. The information about the robot and human positions, orientations and the 3D state of the environment is continuously updated in our 3D representation and planning platform, Move3D [19], which also facilitates our robots to check intra-body and inter-body collisions for itself and for the human model. The robot uses kinematic structures of the agents and performs various virtual actions until the joint limits of the neck and/or torso are reached or the collision of the torso of the agent with the environment is detected.

\subsection{Mightability Maps: Mightability Analyses at 3D Grid Level}

In [16] we have enabled our robot to perform such Mightability Analyses at 3D grid level, which we term as Mightability Map. For continuity we will briefly present the idea with the generalized extension of the concept by implementing it on a different kind of robot as well as with various complementary results and remarks.

\subsubsection{Spatial-Reach Mightability Maps}

One can choose to have a rigorous estimation of reachability, but for the quick computation and updating online, we have chosen the length of the hand tip from the shoulder in a given posture of the agent as the first level of estimation of reachability. This is again inspired from human movement and behavioral psychology $[1,17,18]$, which suggest that the prediction to reach a target with the index finger depends on the distance of the target relative to the length of the arm. Although such prediction deviates by $10 \%$ from the actual arm length, it plays as a key component in actual movement planning. For the sake of not underestimating the reachability, the robot slightly overestimates, which could be further filtered out at later states of planning or decision-making by rigorous testing, where the search space would have already been reduced significantly.

In [16] we have explained how the virtual actions associated with a particular state are applied on the 3D model of the agent to calculate the reachability of the 3D grid cells. Here we will discuss few complementary results and interesting remarks. Spatial-reach Mightability Maps for Human and Humanoid Robot HRP2 from their current state have been shown in 3D, fig. 3(a), and on table plane, fig. 3(b). Robot also distinguishes among the cells, which could be reached only by left hand (yellow), right hand (blue) and by both hands (green). Hence the robot is aware that there is no common reachable region if neither of them will lean for- 


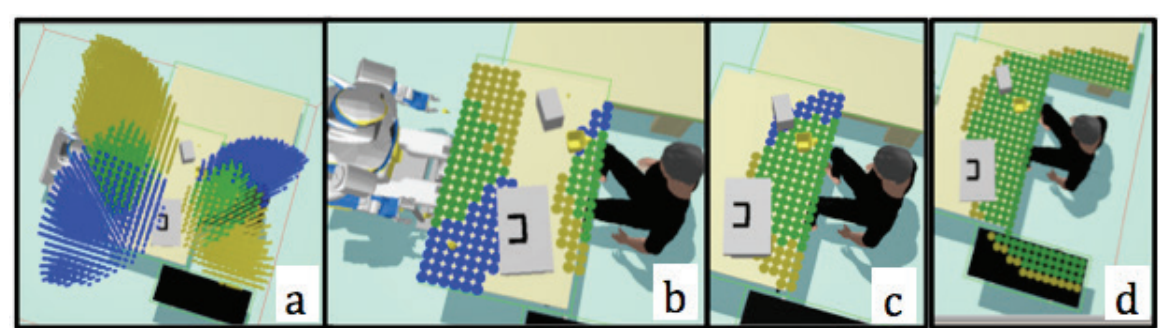

Fig. 3 (a)-(d) (a) Spatial-reach Mightability Map for HRP2 \& Human from their current states in 3D and (b) on table surface, (c) leaning forward reach (d) turnaround and leaning reach, human can reach some parts of the side by table, which are of different heights.

ward. Fig. 3(c) shows reachability of human on table with maximum possible leaning forward. The robot also perceives that if human will turn around and lean he might be able to reach parts of the side-by tables as well, as shown in fig. 3(d).

In [16] we have developed set representation of Mightability Maps and shown its use in finding common mightabilities by various set operations. Such sets not only contain information about what an agent might be able to 'see' or 'reach' but also what the agent might not be able to 'see' or 'reach' from a particular state.

We have extended the concept of Mightability for different type of robots including the single-arm mobile robot Jido. Fig. 4 (a) shows the common reachable region in 3D obtained by intersection operation on spatial-reach Mightability Map of both Human and Jido from their current state. This in fact could serve as candidate place where Jido can hand over an object to the human. As shown in fig. 3(a) there was no common reachable region from the current states of Human and HRP2, but as shown in fig. 4(b) HRP2 is able to estimate that if the human puts effort to lean forward then there might exist a common reachable region. Fig. 4(c) $\&$ (d) show the common reachable region in $3 \mathrm{D}$ and on table plane from the current states of the Human and HRP2 in a different setup where both are sitting sideby-side. These regions respectively could serve as the candidate places to give an object and to put an object for the human to take.

Fig. 5 shows an interesting observation about leaning forward reach. The reachable region by leaning forward in fig. 5(a) is less compared to that of fig. $5(\mathrm{~b})$, even the human is closer to the table in the former case. This is because, as

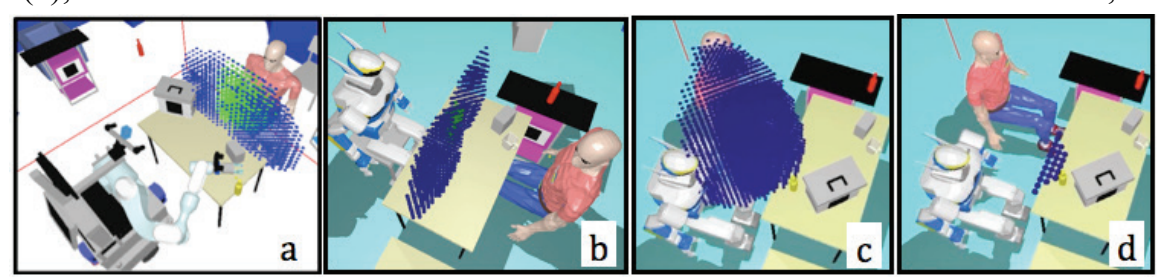

Fig. 4 (a)-(d) Common Mightability (a) Common reachable region in 3D for human and another mobile robot Jido, having one arm, from their current states, (b) common reachable region in 3D from current state of HRP2 and leaning forward state of human, (c) common reachable region for human and HRP2 from their current states, sitting side-byside in $3 \mathrm{D}$ and (d) on table plane. 
Fig. 5(a)(b) Reachability of human on the table surface in leaning forward state. (a) Human closer to the table could lean less compared to (b) sitting away from the table.

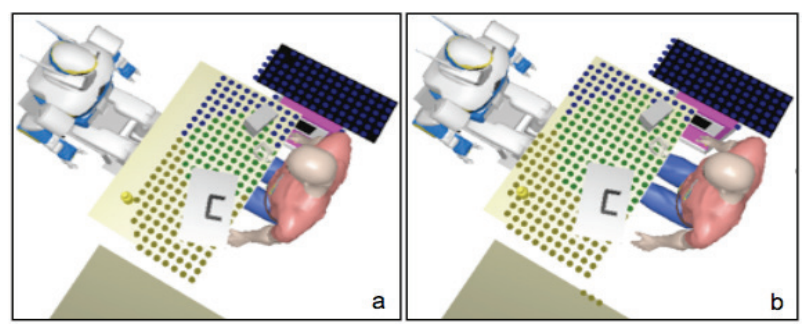

mentioned earlier our approach respects the postural and environmental constraints, and in the former case the human is very close to the table edge hence could lean less as compared to the later case where there is sufficient gap between human torso and the table to lean more without collision.

\subsubsection{Visuo-Mightability Maps}

Robot performs the virtual actions of table 2 on the agents to calculate the $3 \mathrm{D}$ grid based Visuo-Mightability. The approach, which we have described in [16] is based on ray tracing from the eye/camera of the agent and marking the free cells until the first obstacle cell as visible and the cells beyond as invisible.

Red circles in fig. 6(a) show the visibility of the human from his current state on the table plane. The blue cells in fig. 6(b) show the visibility in 3D for HRP2 from its current state. Fig. 6(c) shows the current visibility (green cells) as well as

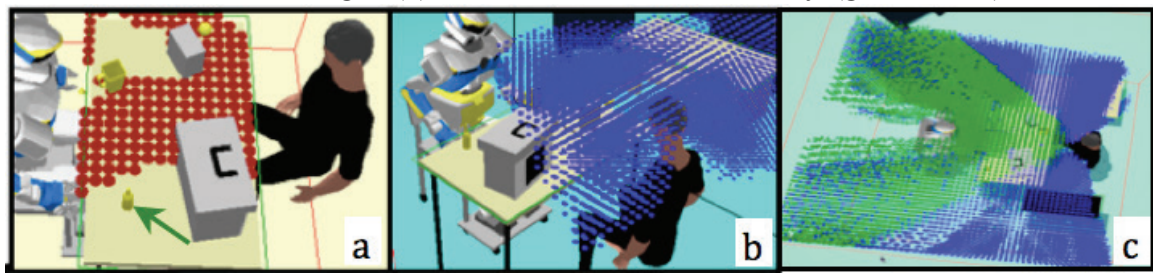

Fig. 6(a)-(c) Different Visuo-Mightability Maps (a) Current visibility of human on table surface and (b) of HRP2 in 3D (c) Current (green circles) and turning the head around (blue circles) visibility of human without turning torso.

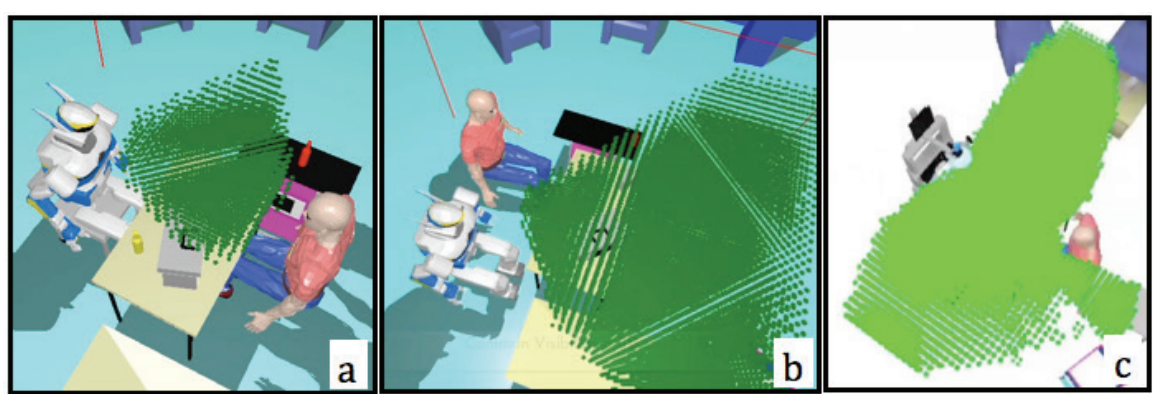

Fig. 7(a)-(c) Common Visibility from the current states of human and HRP2 in face-toface (a) and side-by-side (b) sitting configurations. (c) Common visibility for different robot Jido and human for their current and looking left \& looking right states. 
the visibility when human will turn his head without turning the torso (blue cells).

Fig. 7(a) \& (b) show the common visibility of HRP2 and human in 3D from their current states in face-to-face and side-by-side sitting configurations. Fig. 7(c) shows the common visibility of Jido robot and the human from their current, looking left and looking right states.

Fig. 8 shows an interesting observation. Fig. 8(a) shows the visibility of Human on the table plane from sitting position, whereas fig. 8(b) shows the visibility calculated by the robot by virtually putting the human in standing state. Hence the robot is able to predict that if the human will standup, he might be able to see more regions, shown by blue ellipses, including the region behind the small box, which is currently invisible to him.

Fig. 8 (a)(b) Visibility of human on the table plane (a) from his current state and (b) from standing state. Robot predicts that if human will stand up he might be able to see more regions (shown by blue ellipses), which are currently hidden for him, including the regions behind the small box.

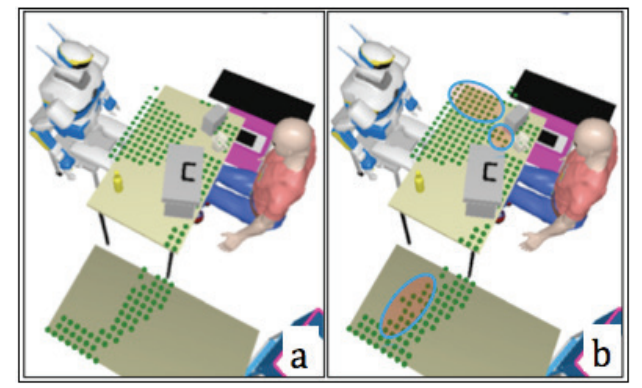

\subsection{Object Oriented Mightability Analyses (OOM)}

We have equipped our robots to perform all the Mightability Analyses at the level of objects also, which we termed as Object Oriented Mightability (OOM). In fact such $O O M$ analyses will serve as complementary information to the $3 \mathrm{D}$ grid level Mightability for various higher-level decision-making and task performing. For example with $O O M$ robot knows which objects are reachable/unreachable and visible/invisible to the agent in a particular state and will use Mightability Maps $(M M)$ to find the candidate places where to perform a particular task like show an object, make an object accessible or even hide an object from the human.

Similar to the $M M$, the robot calculates $O O M$ for all the states shown in table 1 and table 2. An agent can show the reach action for a range of tasks such as, to grasp, to touch, to push, to put something into, etc. Also the answer to the question of how much visible is considered as "object is visible" depends upon the task and the context. Hence again, as first level of estimation, we have chosen the approach to roughly estimate the visibility and reachability of the objects for the sake of fast computation and updating online. This could further be filtered out with rigorous testing at the later stages of actual decision making and planning.

At the first level, the reachability and visibility of an object is estimated based on whether at least one cell (dimension $5 \mathrm{~cm} \times 5 \mathrm{~cm} \times 5 \mathrm{~cm}$ in current implementation) belonging to the object is reachable or visible to the agent or not. Further- 
more robot also keeps track of how many cells belonging to the object are reachable and visible. Hence robot continuously updates the set of Object Level Mightabilities in the form of <agent, state, object, reachable, cell_count $>$ and <agent, state, object, visible, cell_count $>$.

Although we have dedicated modules for rigorous testing for reachability and visibility from a particular state of the agent based on inverse kinematics and pixel level analysis respectively, we prefer to impose such checks at the appropriate later stages of planning and decision making where the search space has already been reduced significantly. However one can use such rigorous testing at the very first level of finding Object Oriented Mightabilities.

The robot performs similar higher level reasoning about common visible and reachable objects in different virtual states of the agents as it does for places in $3 \mathrm{D}$ in the case of Mightability Maps.

\section{Potential Applications and Experimental Results}

The multi-state multi-agent visuo-spatial analyses presented above at 3D grid level (Mightability Maps) and at object level (Object Oriented Mightability) could enrich the robot's reasoning capabilities for a variety of applications. We will briefly discuss few such applications below.

\subsection{Mighability based Framework: 'Finding' Search Space}

We have presented a basic framework in [16], for performing various cooperative as well as competitive tasks like show an object to the human, make an object accessible to the human, hide an object from the human, etc. The framework, based on various task dependent operations on Mightability Maps, significantly reduces the search space for finding the feasible solution for a particular task. Then it assigns weight in the candidate search space. The framework also allows delaying or introducing various constraints and rigorous feasibility tests at appropriate stages of finding the solution, when the search space has been significantly reduced. We have adapted the same framework for a different task of giving an object to the human and that too by a different robot Jido by incorporating additional constraints on grasp and orientation on the object. We will briefly explain the basic framework in this new context.

Fig. 9 shows different steps of the framework. For the current scenario as shown in fig. 10(a), the task is to give the small bottle, shown as red arrow, to the human. Robot is having the set of all the Mightability Maps of itself and of the human. This set is further reduced by the decision on mutual comfort balancing, step (b) of fig. 9. Such comfort balancing needs separate investigation, but for our 


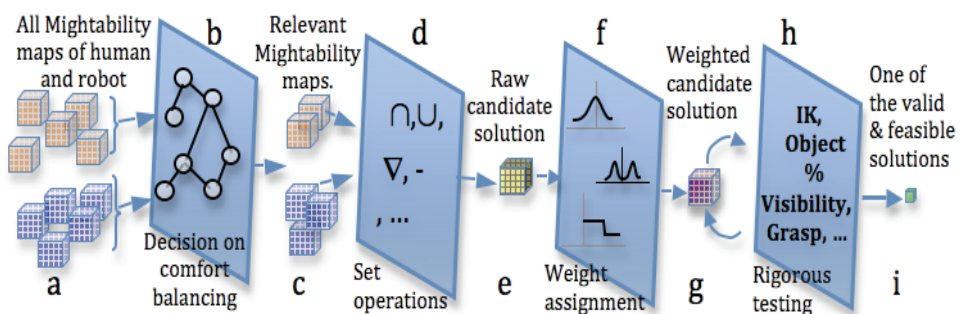

Fig. 9 Mightability Analysis based framework for finding a solution: (a) Initial Mightability Maps, (b) Decision making on relevant Mightability Maps depending on task and required comfort level of agents, (c) relevant Mightability Maps, (d) task specific set operations, (e) raw candidate solution set, (f) weight assignment based on spatial preferences, (g) set of weighted candidate points, (h) applying rigorous and expensive tests on reduced search space, (i) the feasible solution of highest weight.

purpose we assume that the robot is co-operating with the human partner and it does not want the human to stand up or even turn around for taking the bottle. Furthermore it wants to give the object at the place, which is visible to the human from his current state, in other words human need not turn his head left or right to see the object. Hence in step (c) robot gets spatial-reach Mightability Maps, only for the current and leaning forward states of the human, and visuo-Mightability Map for the current state of human. As the robot does not put any restriction on its comfort, it will use all the Mightability Maps of itself. Then depending upon the task, step (d) performs various set operation on these Mightability Maps to find the candidate set of points for performing the task. In step (e), robot gets raw candidate solution space after such operations. The green cells in fig. 10(b) show the raw candidate solution for the current task of giving an object to the human. These are basically the cells, which are commonly reachable by both Human and the Robot and visible by the human in his current state.

Step (f) assigns various weights on this candidate space. Such weight assignment also needs separate investigation on the human preferences, but for the current purpose we will assign a weight by normal distribution centered at a particular distance from the human along the human's current axis. Fig. 10(c) shows the weighted candidate points obtained in the step $(\mathrm{g})$. At this step it is interesting to

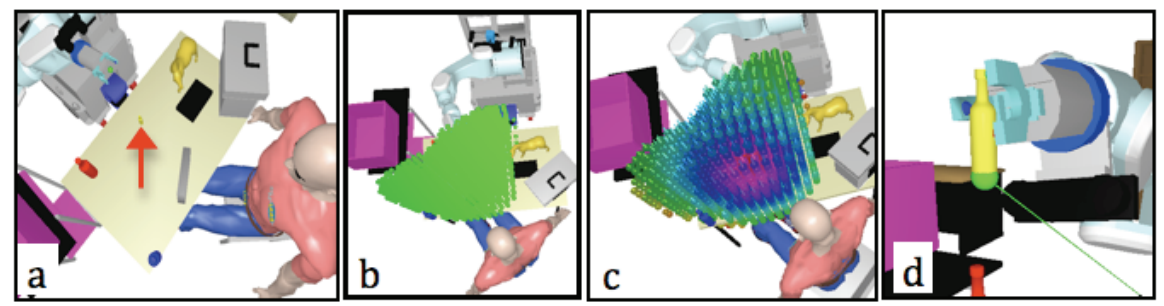

Fig 10 (a)-(d) (a) Initial setup for the task of giving the small yellow bottle, shown by red arrow, to the human (b) raw candidate search space (c) weighted candidate search space for rigorous testing (d) human's perspective when the robot is holding the object to give it to human after finding the feasible solution of highest weight. 
note that the search space has been significantly reduced to 608 cells as compared to $1,80,000$ cells of entire workspace. (The workspace has dimension $3 m \times 3 m x$ $2.5 \mathrm{~m}$ for the current scenario, which has been divided into grid cells of size $5 \mathrm{~cm} x$ $5 \mathrm{~cm} \times 5 \mathrm{~cm}$ ). Now in the step (h) robot puts the bottle virtually at each candidate cell sequentially from highest to lowest weight and introduces various rigorous testing on pixel level visibility of the object, inverse kinematics, grasp by human, etc. Such tests would have been very expensive if done for entire workspace. In our current implementation the first highest weighted candidate cell, where the placement of the bottle satisfies all the feasibility tests is accepted as the feasible solution of highest weight, as shown in step (i). Fig. 10(d) shows the human's visual perspective after the robot has executed the task by carrying and holding the object at the feasible place of highest weight to give it to the human.

For experiments, 3D map of the static environment is provided to the robot. Acquisition of the dynamic objects such as the big and small boxes and the cup is done by stereo-vision based tag identification system through robot's camera. For tracking the human and the object to be manipulated, markers based motion capture system is used. We filter out data of those markers, which are not in the field of view of robot, to avoid the impression of bird's eye view. 3D model of the environment is updated in real time in our integrated 3D representation and planning platform, Move3D [19]. Human gaze is simplified to the human head orientation and the length of human arm is fix in the current implementation. Mightability Maps (MM) and Object Oriented Mightabilities (OOM) are updated online.

Fig 11 shows tasks of showing an object to the human and hiding an object from the human being performed by Humanoid robot HRP2 using the presented framework. Fig. 12 shows tasks of making an object accessible and hiding an object performed by the different mobile robot Jido by using the same framework.
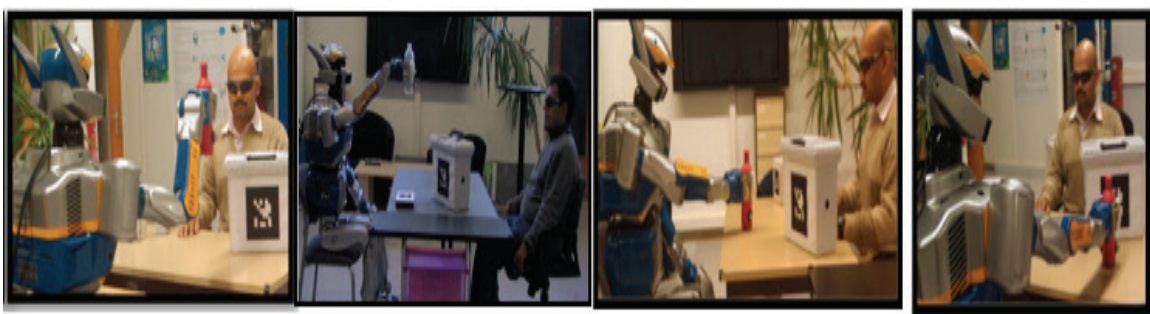

Fig. 11 (a)-(d) (a) and (b) HRP2 showing the bottle to two different humans, (c) reaching and grasping the bottle and (d) hiding it from the human.

\subsection{Predicate for Learning Semantics of Tasks}

For the above experiments the 'meaning' in terms of 'effect' to be achieved for a particular task was already provided to the robot. But such Mightability Analyses could also be used as predicates for learning semantics of various tasks. We 


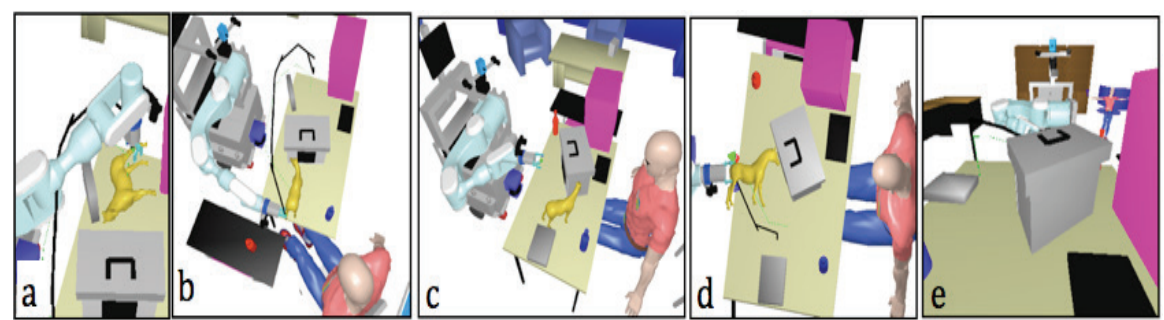

Fig. 12 (a)-(e) (a) initial position of the yellow toy horse for the task of making it accessible to the human, (b) Jido makes the object accessible to the human, (c) initial position of the toy horse which is visible to the human from his current state, (d) Jido is hiding the object from the human, (e) final state of the world from the human's perspective after robot hides the toy horse from the human.

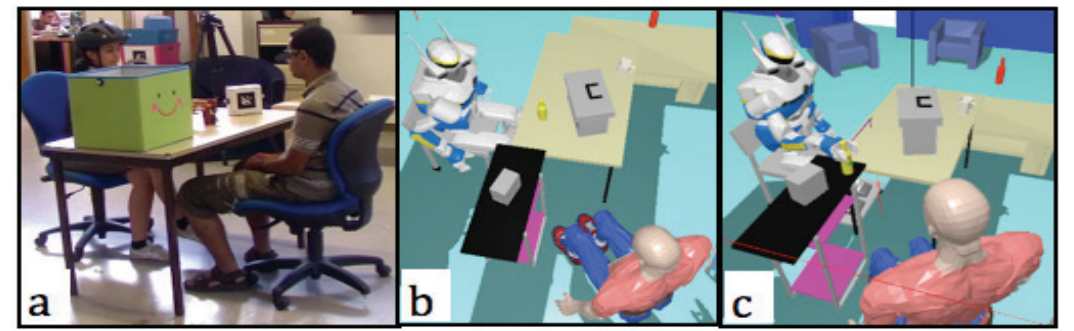

Fig. 13(a)-(c) (a) Collecting data from human performing the task of making an object accessible to the other human. (b) Initial scenario and (c) the final placement of the object for performing the task of making the object accessible learnt by HRP2.

collect data of one person performing a particular task for another person through motion-capture system, as shown in fig. 13(a). We provide the positions of humans and object before and after finishing the task and the name of the task as input to the robot. Although the development of the algorithm is in its initial stage, by analyzing the 'effect' in terms of visibility and reachability of the object from the perspectives of both the humans, robot learns the postconditions to be achieved for that task. For example, for the task of making the object accessible to the human, robot associates the postconditions that it should be visible and reachable by the current state of the target human. Hence the robot is not bound to learn/store the absolute or relative position of object or the trajectory for a task.

Although the data for learning was from the experiments of humans sitting face-to-face, fig. 13(a), robot is able to perform the task of making the bottle accessible in a different setup, fig. 13(b), where the human and robot are not face-toface. Note that as shown in fig. 13(c), the final table to put the bottle for making it accessible to the human is also different from the table at which initially the bottle was placed. This preliminary result just shows the potential use of Mightability Analyses. A separate investigation is desirable on the preconditions, postconditions and the relative weights and preferences, from the learning point of view. 


\subsection{Generation of Shared Plan}

The presented Mightability Analyses could also be used for generating the shared plan for achieving a higher-level task. Fig. 14 shows the initial scenario for the task: "clean the table", by putting the objects into trash bins with the constraints that all the bottles (marked by green arrow) should be in Pink trash bin and all the tapes (marked as red arrow) should be in Blue trash bin. Although separate investigation of various factors in generation of shared planned is required, below we show the preliminary result. It show the potential use of Mightability

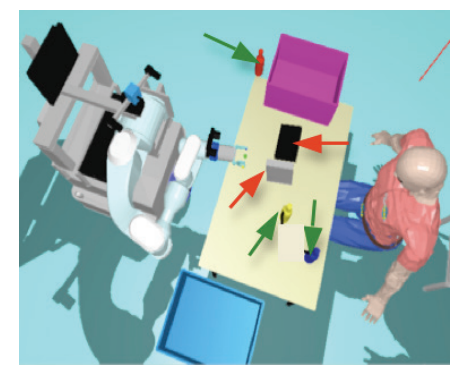

Fig. 14 Scenario for generating the shared plan for the task of cleaning the table. analyses in allocating sub tasks, explaining the reasons behind a decision and proposing co-operative actions if required. The shared plan generated by the robot, for the situation where the robot will try to perform maximal part of task, is as follows:

- I (JIDO) will put RED_BOTTLE in Pink Trash bin, because I know you cannot do it from sitting state. - I (JIDO) will put GREY_TAPE in Blue Trash bin, because I know you cannot do it from sitting state. - I (JIDO) will put YELLOW BOTTLE in Pink Trash bin, although I know you can also do it. • I (JIDO) will put BLUE_BOTTLE in Pink Trash bin, although I know you can also do it. - We need help/co-operation for putting BLACK_TAPE in Blue Trash bin, because none of us can do it alone. Because Blue Trash bin is not reachable by you to put into and BLACK_TAPE is not graspable by Me (JIDO).

Co-operative action proposed by Robot:

- I know you Might be able to give the BLACK_TAPE to Me (JIDO) and Blue Trash bin is reachable by Me (JIDO). So, give the BLACK_TAPE to me and I can put BLACK_TAPE into the Blue Trash bin.

\subsection{Grounding Environmental Changes to Agents and Actions}

Such Mightability Analyses when performed for all the agents in the shared workspace could also be used for predicting the agent who might have caused some changes or who might do some changes (willingly or accidently) in the environment. Furthermore the robot can also predict the associated action/effort by the agent. For example fig 15 shows the leaning forward reachabilities of HRP2 and of both the humans superimposed altogether on the table plane. Note that there are some regions, which are not reachable by any agent from their sitting positions. 
Hence if an object needs to be displaced or has already been displaced about which the robot was oblivious, the robot can predict probabilistically the agent and the associated effort (such as the agent has to stand up and lean) for making that change. Such analyses could also help in multi-agent task allocation as well as action recognition.

\section{Conclusion and Future Works}

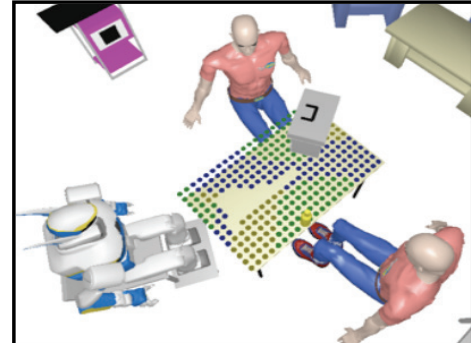

Fig. 15 Leaning forward reachability for HRP2 and both humans superimposed altogether on the table plane.

We have presented the concept of Mightability, which stands for Might be able to, as a multi-state visuo-spatial reasoning for higher level decision making by robot. Our robot performs such analyses for itself as well as for human partner. We have proposed two complementary levels of such Mightability analyses: at 3D grid level, which we termed as Mightability Maps (MM) and at object level, which we termed as Object Oriented Mightability (OOM). We have shown the generalization of the concept with two different types of robots performing various tasks: Humanoid Robot HRP2 and single-arm mobile robot Jido.

Such Mightability analyses could not only be used for decision making which requires analyses of common abilities of the agents but also for the problems which demand what an agent might not be able to do.

Apart from the pointers mentioned in the potential applications, the future works will also focus on its use for bridging the gap between the symbolic and geometric planner, for prediction of human intention and intended action, to enhance the verbalized interaction with the human partner, etc. It would also be interesting to develop methods, which enable the robot to autonomously distinguish between the Mightability analyses, need be done periodically and need to be done specifically in a particular situation. Furthermore such analyses could be extended for more abilities as well as for more states. All these will lead towards a better proactive behavior of the robot in human centered environment.

\section{References}

[1] P. Rochat, "Perceived reachability for self and for others by 3- to 5-year-old children and adults, "Journal of Experimental Child Psychology, vol. 59, Issue 2, April 1995, pp. 317-333.

[2] A. J. Caron, E. J. Kiel, M. Dayton, and S. C. Butler, "Comprehension of the referential intent of looking and pointing between 12 and 15 months," Journal of Cognition and Development, vol. 3(4), 2002, pp. 445-464. 
[3] S. Dunphy-Lelii, and H. M. Wellman, (2004) "Infants' understanding of occlusion of others' line-of-sight: Implications for an emerging theory of mind," European Journal of Developmental Psychology, vol 1(1), 2004, pp. 49-66.

[4] G. O. Deak, R. A. Flom, and A. D. Pick, "Effects of gesture and target on 12-and18-montholds' joint visual attention to objects in front of or behind them," Developmental Psychology,.36(4), 2000, pp. 511-523.

[5] H. Moll, and M. Tomasello, "12-and18-month-old infants follow gaze to spaces behind barriers," Developmental Science, vol. 7(1), 2004, pp. F1-F9.

[6] F. Zacharias, Ch.Borst and G. Hirzinger, "Capturing robot workspace structure: representing robot capabilities," in Proceedings of the IEEE/RSJ IROS, pp. 3229-3236, San Diego, USA, October, 2007.

[7] F. Zacharias, Ch.Borst and G. Hirzinger, "Online Generation of Reachable Grasps for Dexterous Manipulation Using a Representa tion of the Reachable Workspace," in Proceedings of the International Conference on Advanced Robotics, Germany, 2009.

[8] L. Guilamo, J. Kuffner, K. Nishiwaki and S. Kagami, "Efficient prioritized inverse kinematic solutions for redundant manipulators," in Proceedings of IEEE/RSJ IROS, pp. 1905-1910, 2005.

[9] Y. Guan and K. Yokoi, "Reachable Space Generation of A Humanoid Robot Using The Monte Carlo Method," in Proceedings of IEEE/RSJ IROS, pp. 1984-1989, Oct. 2006.

[10] C. Breazeal, M. Berlin, A. Brooks, J. Gray, and A. L. Thomaz, "Using perspective taking to learn from ambiguous demonstrations," Robotics and Autonomous Systems, pp. 385-393, 2006.

[11] M. Johnson, and Y. Demiris, "Perceptual Perspective Taking And Action Recognition," International Journal of Advanced Robotic Systems, Vol.2(4), Dec. 2005, pp 301-308.

[12] J. Gregory Trafton, Nicholas L. Cassimatis, Magdalena D. Bugajska, Derek P. Brock, Farilee Mintz, Alan C. Schultz, "Enabling effective human-robot interaction using perspective-taking in robots", IEEE Transactions on Systems, Man, and Cybernetics, 2005, 460-470.

[13] L. F. Marin-Urias, E. A. Sisbot, A. K. Pandey, R. Tadakuma, and R. Alami, "Towards shared attention through geometric reasoning for human robot interaction," IEEE-RAS International Conference on Humanoid Robots, pp. 331-336, Paris, France, 2009.

[14] D. L. Gardner, L. S. Mark, J. A. Ward, and H. Edkins, "How do task characteristics affect the transitions between seated and standing reaches?," Ecological Psychology, vol. 13, 2001, pp. $245-274$.

[15] H. J. Choi, and L. S. Mark, "Scaling affordances for human reach actions," Human Movement Science, vol. 23, 2004, pp. 785-806.

[16] A. K. Pandey and R. Alami, "Mightability Maps: A Perceptual Level Decisional Framework for Co-operative and Competitive Human-Robot Interaction", IEEE/RSJ International Conference on Intelligent Robots and Systems (IROS), pp. 5842-5848, Taiwan, 2010.

[17] C. Carello, A. Grosofsky, F. D. Reichel, H. Y. Solomon and M. T. Turvey, "Visually Perceiving what is Reachable," Ecological Psychology, vol. 1(1), 1989, pp. 27-54.

[18] R. J. Bootsma, F. C. Bakker, F. J. Van Snippenberg, and C. W. Tdlohreg, "The Effects of Anxiety on Perceiving the Reachability of Passing Objects, "Ecological Psychology, Vol 4(1), 1992, pp. 1-16.

[19] T. Simeon, J.-P. Laumond, and F. Lamiraux, "Move3d: A generic platform for path planning," in IEEE International Symposium on Assembly and Task Planning, Fukuoka, Japan, 2001, pp. 25-30. 